\title{
Parallel and serial search in haptics
}

\author{
K. E. OVERVLIET, J. B. J. SMEETS, AND E. BRENNER \\ Vrije Universiteit Amsterdam, Amsterdam, The Netherlands
}

\begin{abstract}
We propose a model that distinguishes between parallel and serial search in haptics. To test this model, participants performed three haptic search experiments in which a target and distractors were presented to their fingertips. The participants indicated a target's presence by lifting the corresponding finger, or its absence by lifting all fingers. In one experiment, the target was a cross and the distractors were circles. In another, the target was a vertical line and the distractors were horizontal lines. In both cases, we found a serial search pattern. In a final experiment, the target was a horizontal line and the distractors were surfaces without any contours. In this case, we found a parallel search pattern. We conclude that the model can describe our data very well.
\end{abstract}

Visual search experiments have been very successful in providing insight into how visual information processing works. Much less is known, however, about haptic information processing. Haptic search may help us find out how haptic information is processed.

Lederman and Klatzky (1997) did several search experiments in the haptic domain. They investigated which haptic properties are available for processing relatively early after initial contact, by presenting different kinds of stimuli to their participants' fingertips. They divided their stimuli in four dimensions: First, they tested the material dimension, including the roughness, hardness, and warmness of the material. Second, they tested "abrupt surface discontinuities," such as finding a raised bar among flat surfaces or searching for a deep hole between shallow holes. Third, they tested relative orientation, since the target had a different orientation from the distractors. Finally, they investigated continuous 3-D surface contours, with a difference in slant or curvature between the target and the distractors. The material and the abrupt surface discontinuities produced low search function slopes, indicating more or less parallel search. Relative orientation and continuous 3-D surface contours, on the other hand, produced relatively steep slopes, indicating serial search.

However, Lederman and Klatzky's (1997) results do not match those typically found in visual search experiments. For example, in the target-absent conditions, Lederman and Klatzky did not find a slope that was twice as high as the target-present slope (cf. Treisman \& Gelade, 1980). They also did not find similar search times for target-present and target-absent trials when there was only one item in the display, as is generally found in visual search (Mori \& Kataoka, 2004; Saarinen, 1995; Treisman \& Gelade, 1980).

Is haptic search different from visual search? The way of perceiving is certainly different; in haptic search, the number of stimuli presented is generally the same as the number of sensors (the fingertips), and in visual search, different numbers of stimuli are presented to at most two sensors (the retinas of both eyes). Are the underlying processes also different? In visual search, increasing the number of items in a display increases the time it takes to detect a target, unless the target has a characteristic feature that the other items do not have (Treisman \& Gelade, 1980). In the latter case, search time is independent of the number of items. Examining the extent to which increasing the number of items influences detection time can therefore be used to identify what we could consider the characteristic features of vision. If the target possesses a feature that the distractors do not have, the search time is independent of the number of distractors; in other words, the search process is parallel. If the difference between target and distractors is more complicated, each item has to be checked to determine whether the relevant features are present; in this case, the search process is serial.

For example, when one searches for a red pencil between blue ones, the target pencil possesses a characteristic simple feature that the distractors do not possess (the color red), so regardless of how many blue pencils surround it, the target will take about the same amount of time to find. In contrast, searching for one pencil with a broken point between sharpened pencils takes longer, the more the pencils that are present; the target pencil can be found only by serial scanning.

The reasoning above describes the search behavior predicted by Treisman and Gelade's (1980) feature integration theory of visual attention. This theory states that simple features are registered in parallel and that objects are perceived by "gluing" these features together through directed spatial attention. An implicit assumption here is that a display can be described in terms of simple features. If the target differs in simple features from the distractors, the search process is parallel; otherwise, it is serial. There are many examples for which this theory holds in visual perception (see, e.g., Brown, Weisstein, \& May, 1992;

K. E. Overvliet, krista.overvliet@gmail.com 
Joseph, Chun, \& Nakayama, 1997; Julesz, 1984, 1986; Maioli, Benaglio, Siri, Sosta, \& Cappa, 2001; Nakayama \& Silverman, 1986; Palmer, 1994; Quinlan \& Humphreys, 1987; Treisman \& Gormican, 1988; Wolfe \& Horowitz, 2004), but there are some conditions in which its models do not fit precisely, leading to modifications.

An influential alternative is based on stimulus discriminability. In this alternative, the "difficulty of search increases with increased similarity of targets to nontargets and decreased similarity between nontargets, producing a continuum of search efficiency" (Duncan \& Humphreys, 1989). Irrespective of nontarget similarity, as long as the similarity between target and nontarget is sufficiently low, the search time is independent of the array size. Even increasing target-nontarget similarity has relatively little effect when nontarget similarity is maximal (identical nontargets). However, when nontargets match features of the target template, search efficiency will degrade.

Wolfe, Cave, and Franzel (1989) suggested that features are processed in parallel and that the efficiency of the search process is a function of the quality of the guidance provided by the parallel processes. Because of noise in the parallel maps, a serial stage has to examine incorrect items if the differences are not very large. The presence of a unique feature generates a strong signal that quickly exceeds the background noise, and search times are then independent of the number of distractors. Both of these modifications become relevant when it is difficult to distinguish the target from the distractors, so that performance is intermediate between the purely serial and purely parallel predictions.

The value of all such theories is that they can be used to develop quantitative models of human performance. In this article, we propose a model for haptic search with a clear distinction between serial and parallel search patterns. In this model, we assume that we are certain about the relevant units and, therefore, the number of items. In our haptic search experiment, each item has its own sensor (it is presented to a separate fingertip), so determining the number of items is quite straightforward. We consider that this design provides optimal conditions for testing the model's validity. The extent to which such a model can be applied to search in other modalities or under different conditions remains to be seen. We use clearly discriminable stimuli because the distinction between parallel and serial search should be clearest with such stimuli. In the section below, we explain the model.

\section{Model}

Serial and parallel search differ in more than the increase in the time required to find the target when more items are present. They are also associated with different relationships between the reaction times (RTs) in the target-present and target-absent conditions. In serial search, when the target is present, it will be found on average after scanning half of the distractors; the "effective" number of items scanned is thus 1.5, 2.5, and 3.5 for displays of two, four, and six items. In the target-absent condition, all items have to be scanned in order to be sure that no target is present. The effective number of items in that case equals the total number of items. As a result of these facts, the slope of the search function in the targetpresent condition will be half the magnitude of that in the target-absent condition. The resulting search functions are shown in the left panel of Figure 1. The serial search model is a single linear regression if the search time is expressed as a function of the effective number of items, including both the target-present and target-absent conditions. To conform to the tradition in the search literature, we will report the slope for the target-present trials in terms of the total number of display elements for our fit of the serial search model. This slope is by definition half of the slope in the target-absent condition (i.e., half of the slope in terms of the effective number of items). This results in the following search functions with slope $s$ (i.e., the increase in time per item) and intercept $t_{1}$ (i.e., the time for one item). These parameters are the same for both the
Serial Search

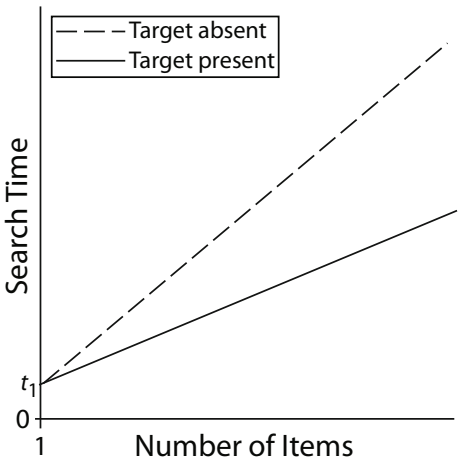

Parallel Search

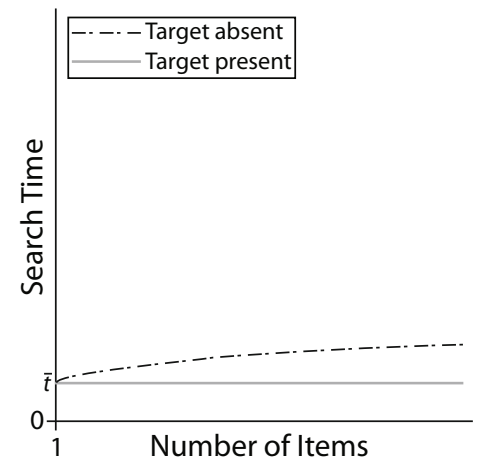

Figure 1. Haptic search models for serial and parallel search. The left panel shows the predictions of a serial search model with arbitrary values of $t_{1}$ (intercept) and $s$ (slope when target present). The slope of the target-absent function is $2 s$. The right panel shows the predictions of a parallel search model for an arbitrary $\bar{t}$ and $\sigma$. At the intercept (i.e., at $x=1$, since one item is in the display), the model predicts equal search times in the target-present and -absent conditions for both kinds of search. 
target-present (Equation 1) and target-absent (Equation 2) conditions:

$$
\begin{aligned}
& \operatorname{RT}(n)=t_{1}+(n-1) s, \\
& \operatorname{RT}(n)=t_{1}+(n-1) 2 s .
\end{aligned}
$$

In parallel search, the slope of the search function in the target-present condition is 0 , since the search time stays the same, no matter how many items are in the display. The distribution of RTs is then, by definition, the distribution for one finger. However, in the target-absent condition, the search times go up a little, in a nonlinear way, with the number of items because the search time for each finger varies from trial to trial. In the target-present condition, this does not influence the overall search time; the overall average search time equals the average search time of an individual finger, because searching stops as soon as the search time elapses for the finger under which the target is found. In the target-absent condition, this is not the case; to be sure that no target is present, a subject must "wait" until processing has finished for all fingers, whatever is under them. In this condition, the overall search time depends on when processing has finished for the slowest finger.

To fit a parallel search function to the data, we reason as follows: The distribution of the longest RT of $n$ fingers can be found by taking the $n$th power of the cumulative distribution of the times of an individual finger. If we as- sume that the detection times that each finger needs are distributed normally (standard deviation $\sigma$ ) around the median and mean $(\bar{t})$, the median RT of $n$ fingers $[\mathrm{RT}(n)]$ for when a target is present and when it is absent are given by Equations 3 and 4, respectively: ${ }^{1}$

$$
\begin{aligned}
& \operatorname{RT}(n)=\bar{t}, \\
& \operatorname{RT}(n)=\bar{t}+\sigma \sqrt{2} \cdot \operatorname{erf}^{-1}\left[-1+2^{n} \sqrt{0.5}\right] .
\end{aligned}
$$

Thus, for the parallel search model, we need to fit two parameters, $\bar{t}$ and $\sigma$, of which only $\bar{t}$ is relevant for the target-present condition.

The intercept in both serial and parallel search is the average time required to process one item in the display, which depends only on the difficulty of identifying the items presented.

The resulting parallel haptic search function is shown in the right panel of Figure 1. We will next examine whether these models fit haptic search functions and, if so, whether we can distinguish between parallel and serial search.

When we fit the models to the data of a few of the experiments in Lederman and Klatzky's (1997) study, we see that neither the serial nor the parallel model provides a good fit (Figure 2). However, Lederman and Klatzky's pattern of results may partly have been due to their experimental design, in which participants did not know how many items they were going to be shown. The number of
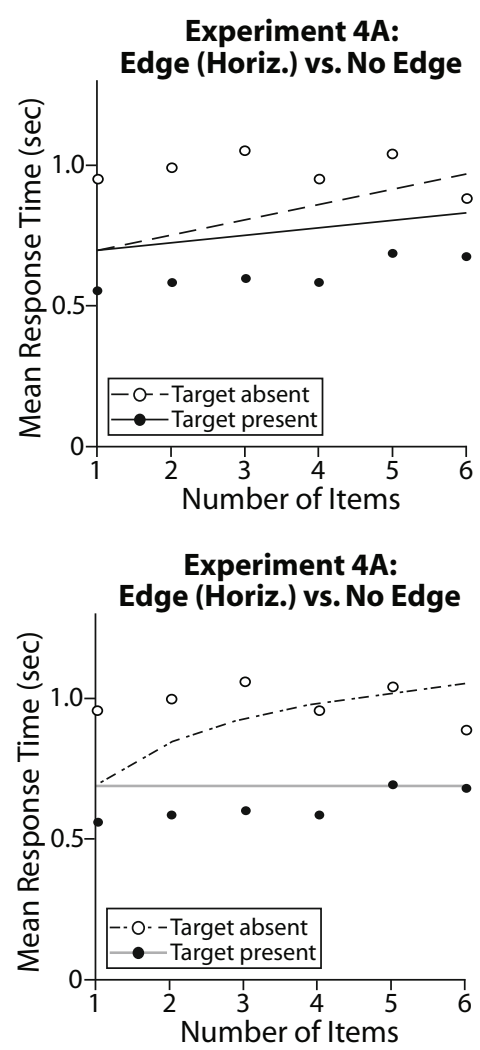
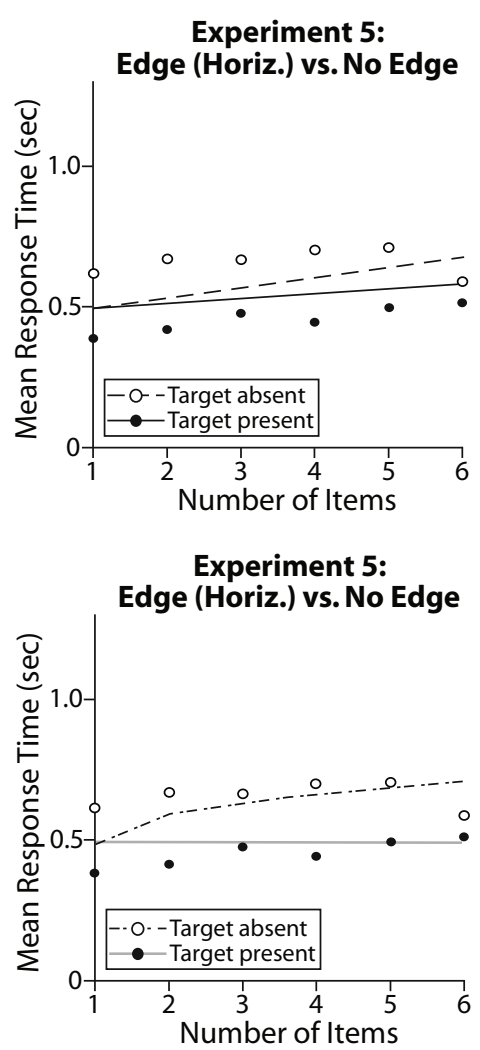
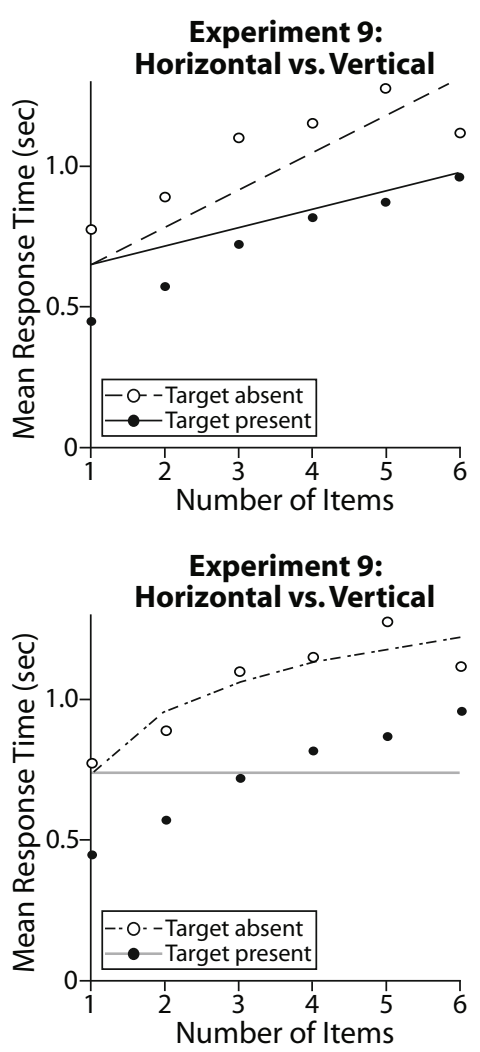

Figure 2. The data of Experiments 4A, 5, and 9 of Lederman and Klatzky's (1997) study. The lines represent the best fits of our serial (top three graphs) and parallel (bottom three graphs) models. 
items varied from trial to trial (from one to six), and the location of the items was not known in advance. Thus, participants had to detect the target when it was present, not only to distinguish it from the other items, but also to determine whether there was an item at each position.

Inspection of Figure 2, in which we plot two conditions from Lederman and Klatzky's study that were interpreted as giving rise to parallel search (detecting an edge among blank units) and one that was interpreted as giving rise to serial search (detecting a vertical line among horizontal ones), reveals that this difference in their methodology may be an issue. The lines in the figure show the best fits of the two sets of equations that we propose. It is evident that neither fits the data very well. In particular, there is a difference in response times even between detecting whether a single target is present or absent. A possible explanation for this difference in the "no edge" condition is that a "blank facet" (empty display element) is equivalent to no edge. The participant therefore first had to determine which fingers were in contact with an item, and then to identify the target between those items. If searching for items between "blank facets" was not completely parallel, we may not be using the relevant units when fitting our model to these data. An obvious solution would be to repeat such experiments, keeping the number of items constant within each block.

In our experiments, we presented different numbers of items in separate blocks to both index fingers; both index and middle fingers; or both index, middle, and ring fingers, so that the participants knew where the items would be. Participants had to indicate target presence or absence by lifting the finger under which they thought the target was present. If they did not find a target, they had to lift all fingers.

\section{EXPERIMENT 1}

In our main experiments, the dependent variables consisted of the search time and the time required to lift the finger. Before we conducted the search experiments, we wanted to check whether all fingers (index, middle, and ring) have about the same mechanical lifting time. Our initial thoughts were that the ring finger would have the slowest lifting time, because subjectively it seems to be harder to lift this finger.

\section{Method}

Participants. Twelve participants, 9 male and 3 female, with a mean age of 31.8 years (range 24-46) participated in this experiment. Two of them stated that they were left-handed.

Stimuli and Apparatus. Six display elements were made of ZY-TEX2 Swell paper by using the ZY-FUSE heater (Zychem Ltd., Cheshire, England). These elements consisted of circles in the paper with a line width of $1.4 \mathrm{~mm}$, which protruded about $1 \mathrm{~mm}$ from the surrounding surface of the paper. They were positioned so that participants could easily put the fingertips of their index, middle, and ring fingers on the centers of the display elements. A sensor under each element measured whether there was a finger on top of it. In order to determine RTs, the sensors were connected to a computer. A curtain was placed between the participant and the apparatus to prevent the participant from seeing the display. The apparatus is shown in Figure 3. The stimulus in this experiment was a $4500-\mathrm{Hz}$ tone.

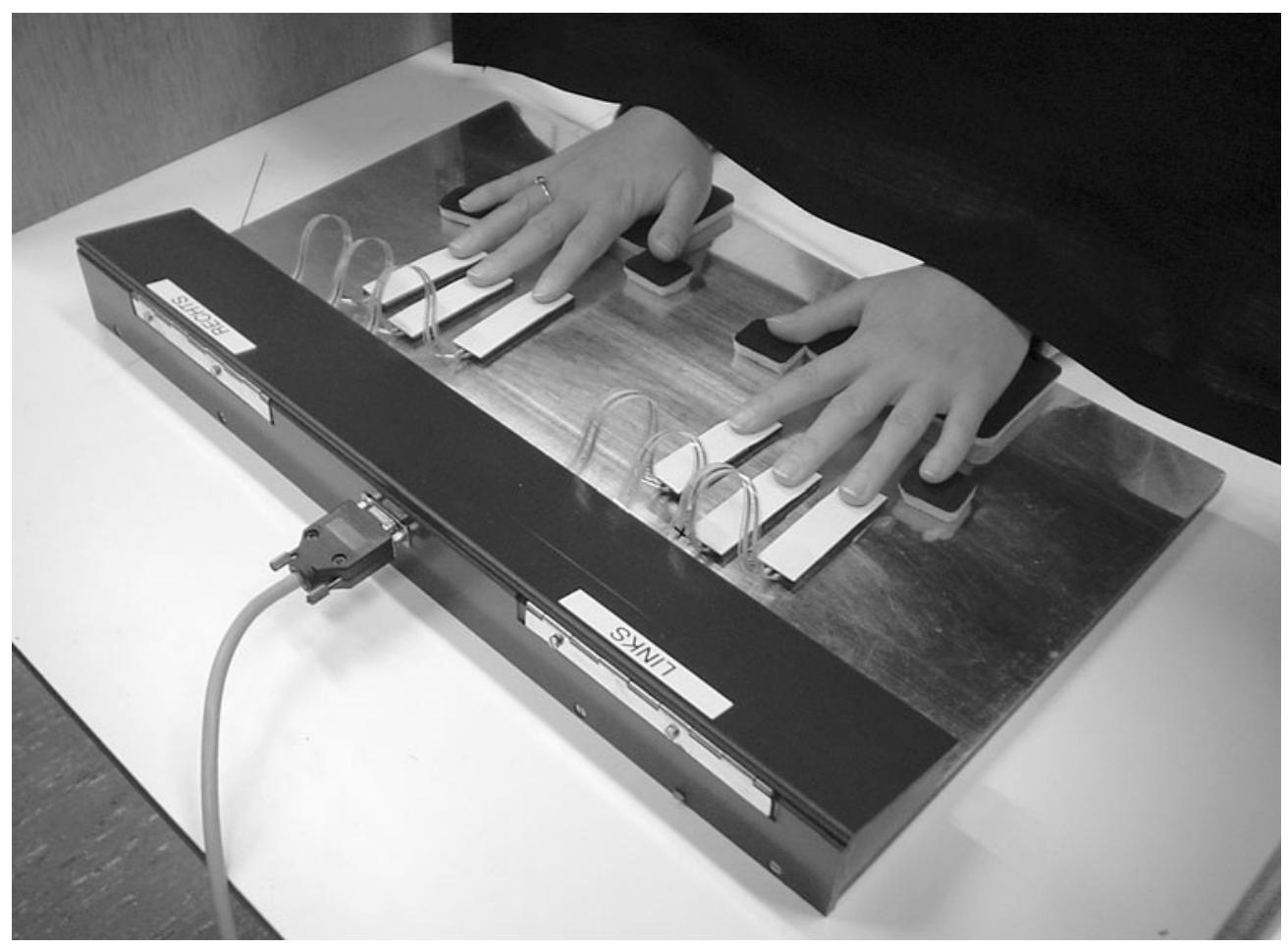

Figure 3. The experimental setup. The positions of the stimulus elements could be adjusted to the positions of the fingers of the participant. Under each element was a sensor that measured whether a finger was on top of it. 
Procedure. Participants put their fingers on the display elements. Their task was to lift one or all of their fingers as soon as possible after hearing the tone. To indicate which finger or fingers the participant had to lift, the experimenter either touched the finger in question or stated that the participant had to lift all fingers. After a random delay, the tone sounded, and the participants had to lift the appropriate finger(s) as soon as possible. There was a single block of 140 trials. Within this block, 20 replications of each of the seven conditions (lifting the index, middle, and ring finger of each hand, or lifting all six fingers) were presented in a random order.

Analysis. RT was defined as the time that elapsed from the moment the tone sounded until the target finger was lifted. In the allfingers condition, the RT was the time that elapsed until the first finger was lifted. We removed all data points below $100 \mathrm{msec}$, because this indicated a nonphysiological RT, for instance caused by bad positioning of the finger on the sensor. We calculated the median RT for each finger of each participant. To see whether there were any differences between the median RTs, we used a univariate ANCOVA with finger as the factor and participant as the covariate.

\section{Results}

The results are shown in Figure 4. The mean overall RT was $263 \mathrm{msec}$. There was no main effect of finger and no effect of participant.

\section{Discussion}

There were no differences in RTs between the different fingers. If anything, the ring finger seemed to be faster than the others. Thus, we did not need to take account of the mechanical lifting time when designing the following experiments.

\section{EXPERIMENT 2}

We designed Experiment 2 to check whether our model would be able to adequately fit the search times if we presented trials with the same number of items in blocks and used a simple stimulus-response compatibility. We chose a very common target-distractor pair for eliciting a parallel pattern in visual search, a cross as a target and circles as distractors (Shen, Reingold, \& Pomplun, 2000). This target differs from the distractors in many ways (line crossings, line endings, closed shape, curvature); we expected the distinction itself to be quite easy to make, so that performance should be either serial or parallel (see the introduction above).

\section{Method}

Participants. Eight participants, 4 male and 4 female, with a mean age of 31.5 years (range 24-46) participated in this experiment. Two of them stated that they were left-handed, and 6 had also taken part in Experiment 1.

Stimuli and Apparatus. The display elements were made of ZY-TEX2 Swell paper and consisted of different figures that protruded about $1 \mathrm{~mm}$ from the surface of the paper. The target was a cross, and the distractors were circles. The size of the figures was about $60 \%$ of the width of the individual participant's index finger, resulting in widths of the stimuli of $8.5,9.6$, or $10.8 \mathrm{~mm}$, with a line width of $1.4 \mathrm{~mm}$. The same apparatus was used as in Experiment 1.

Procedure. We presented haptic stimuli to the fingertips of the ring, middle, and index fingers of both of the participants' hands. The task was to find the target among the distractors. Participants started by lowering two, four, or six of their fingers simultaneously onto the display elements in response to a tone. As soon as they found the target, they had to lift the finger under which they felt the target. If they could not find a target, they had to lift all of their fingers. The participants were told that they should be as fast as possible without making any errors; they were allowed to move their fingers over the stimuli, as long as they always stayed in contact with the same elements. The experiment consisted of three blocks of 40 trials each. The number of items (and of the fingers contacting them) was constant within a block. The items were divided symmetrically between the two hands: In the two-item block, only the index fin-

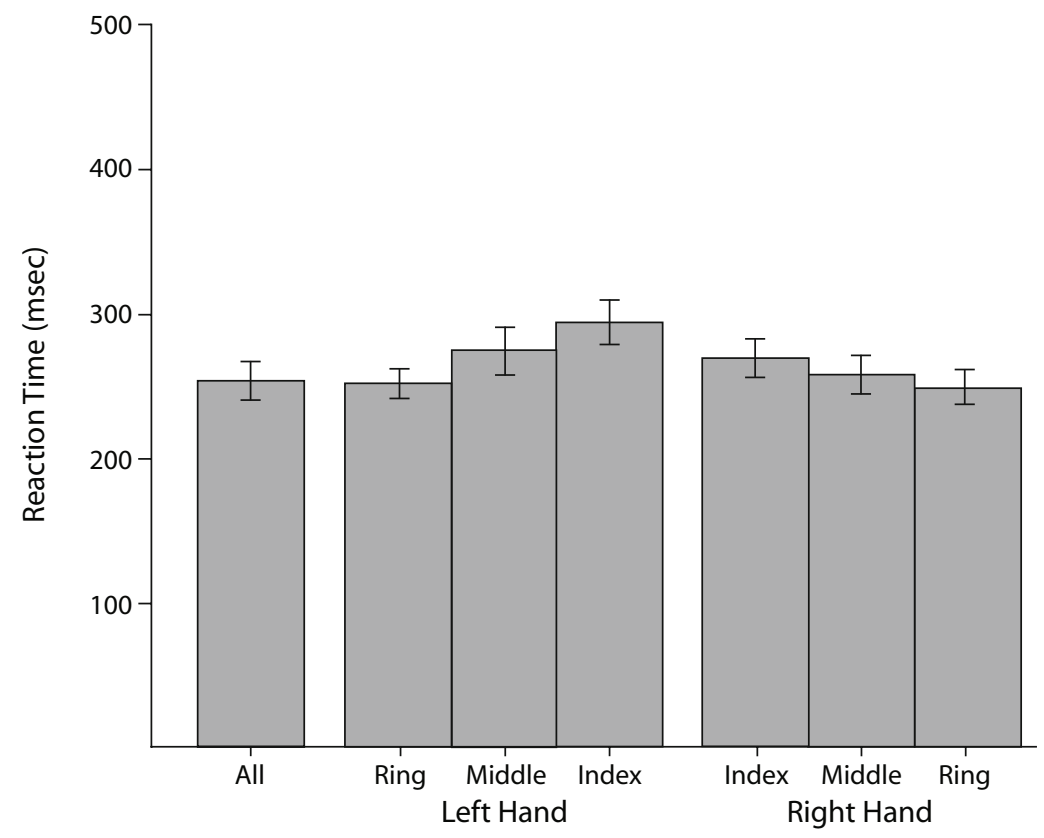

Figure 4. Means of the participants' median reaction times for the seven different conditions in Experiment 1 (with standard errors). 
gers were used; in the four-item block, the index and middle fingers were used; and in the six-item block, the index, middle, and ring fingers were used. Ten of the trials in a block (25\%) had no target, and the other 30 had one target and one, three, or five distractors. The position of the target varied at random between the fingers that had items.

Analysis. We determined the median search times for each participant, condition, and number of items. We discarded search times lower than $100 \mathrm{msec}$ and trials in which a participant made an error. Search time was defined as the time that elapsed from the moment that the first finger touched an element until the moment that the first finger was lifted. Thus, when all fingers were lifted, the time was determined by the first finger to leave the display (as in Experiment 1 and all further experiments).

To test whether there were effects of the number of items in the display and of target presence, we performed a repeated measures ANOVA with two factors: number of items (two, four, or six) and target presence (present or absent). We decided that if there was a significant effect of number of items, we would test whether the data conformed to the serial search model by conducting a linear regression to the average values, as described in the introduction. This regression yielded one slope and one intercept (search time for one element) for both conditions. If the ANOVA did not reveal a significant effect for number of items, we would fit the parallel search model to the data. To test whether the regression model was a valid fit, we used a $\chi^{2}$ goodness-of-fit test (Press, Flannery, Teukolsky, \& Vetterling, 2002). This test gives a measure of the relation between the standard error in the measurements and the deviations from the fit. If $\chi^{2}$ is greater than 1 , the data points were farther from the fit than expected. The $p$ values given below are the probabilities that we should reject the fit.

\section{Results}

The results are shown in Figure 5. We found significant main effects of number of items $(p<.01)$ and target presence $(p<.05)$, and also a significant interaction between the two $(p<.05)$. We also found a linear relation between the effective number of items and search time: The slope of the search model was $290 \pm 54 \mathrm{msec}$ per item; the intercept was $686 \pm 144 \mathrm{msec}$. Overall, the fit was very good $\left(\chi^{2}=0.22, v=4, p<.01\right.$; see the inset of Figure 5$)$

Participants seldom made errors, and if they did make one it was to indicate that there was no target when there was one. They never indicated that a distractor was the target. Thus, discriminating targets from distractors was not an issue for these items (Duncan \& Humphreys, 1989; Wolfe, 1994; Wolfe, Cave, \& Franzel, 1989). The mean error rates were $0.3 \%$ in the two-item condition, $1.5 \%$ in the four-item condition, and $3.1 \%$ in the six-item condition.

\section{Discussion}

Our serial search model fits the data very well, with a slope well above 0 . The fact that search was serial is quite remarkable, since the target " $\mathrm{X}$ " possessed unique features (e.g., the straight lines, intersections, and line endings) that were absent in the distractors. Although this choice of features was based on visual search, and there is no reason to expect the features that yield a parallel search pattern in vision to also do so in haptics, we intuitively considered this kind of information about edges to be very relevant for haptics, and therefore to be processed efficiently. These results confirm Lederman and Klatzky's (1997) findings that this is not the case.

\section{EXPERIMENT 3}

In our first search experiment (Experiment 2), the target differed from the distractors in complex features, such as curvature and intersection. Since this yielded a serial search pattern, we conducted a second, similar experiment to determine whether differing in the most basic spatial aspect of edges, their orientation, would yield a parallel search pattern.

\section{Method}

The same method for measuring and analyzing the data was used as in Experiment 2, and the same participants participated in this experiment. The only difference was that this time a vertical line was used as the target and the distractors were horizontal lines. The lines had a length of $2 \mathrm{~cm}$ and a width of $1.4 \mathrm{~mm}$, so that the full length or width of the finger pad was covered. In all other respects, Experiment 3 was identical to Experiment 2.

\section{Results}

The results are shown in Figure 6. Again, we found significant main effects for number of items $(p<.01)$ and for target presence $(p<.05)$ and a significant interaction $(p<$ $.05)$. We also found a linear relation between the number of items and search time. The slope of the search model was $215 \pm 37 \mathrm{msec}$ per item, the intercept was $493 \pm 101 \mathrm{msec}$ (one item in display), and the fit was very $\operatorname{good}\left(\chi^{2}=0.115\right.$, $v=4, p<.005)$. The average RT was lower than that of Experiment $2(p<.05)$. Again, participants occasionally failed to detect the target but never indicated that the wrong item was the target. The number of errors depended on the number of items $(p<.05)$; the mean error rates were $0.9 \%$ in the two-item condition, $0.6 \%$ in the four-item condition, and $0.9 \%$ in the six-item condition.

\section{Discussion}

The shorter RTs in comparison with those of Experiment 2 show that we succeeded in designing an easier experiment. Once again, we found a serial search pattern, although we used the simplest spatial feature we could think of. Apparently, even line orientation is not sufficient to yield a parallel search pattern in haptic perception, confirming Lederman and Klatzky's (1997) conclusion based on similar targets but different methods (rightmost panels of Figure 2). However, in contrast to their data, ours fit a serial search model very well. In order to examine whether we could also obtain haptic data that fit a parallel search pattern, we designed Experiment 4 on the basis of the assumptions that the poor fit in the lower left and center panels of Figure 2 was a consequence of Lederman and Klatzky's methods (as explained in the introduction) and that the task itself was parallel.

\section{EXPERIMENT 4}

In this experiment, participants had to search for a target between empty display elements. If we were to find a parallel search function, it would mean that people can detect structures in parallel, but not identify them from their spatial features in parallel. If we were to still find a serial search function, it would put our methodology in question, 


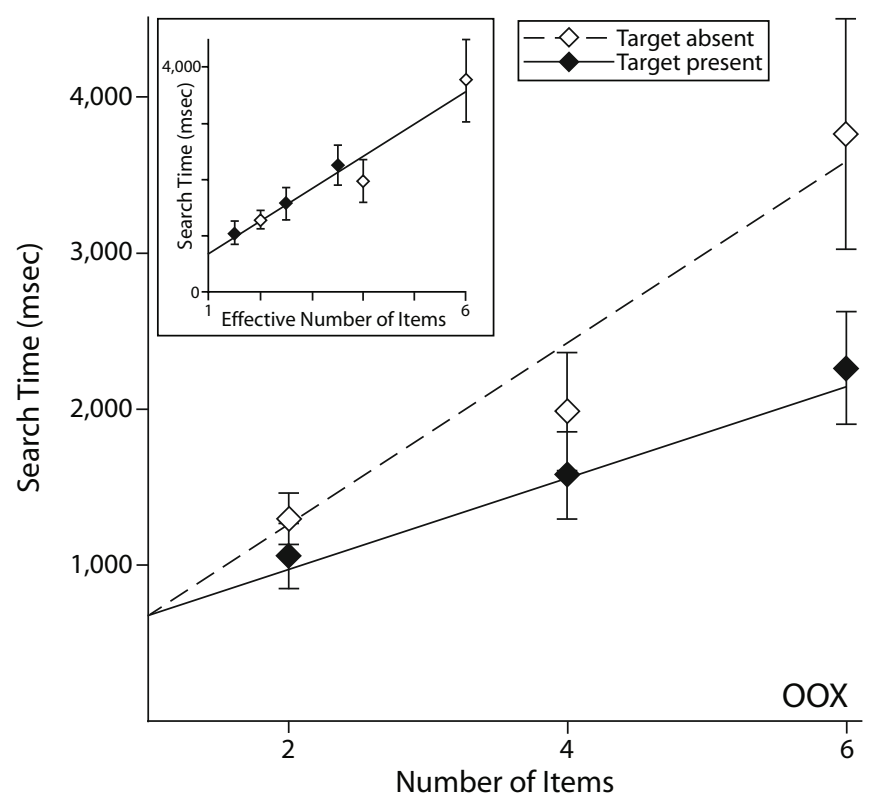

Figure 5. Means of the participants' median reaction times in Experiment 2 (with standard errors). The slope (s) of the serial search model is $290 \pm 54 \mathrm{msec}$ per item. The intercept $\left(t_{1}\right)$ is $686 \pm 144 \mathrm{msec}$. The graph in the inset shows the actual fit of the serial search model to the data. This fit is transformed to the dashed and continuous lines in the main figure (see the Model section for more details). The target (cross) and distractors (circles) are shown in the bottom right.

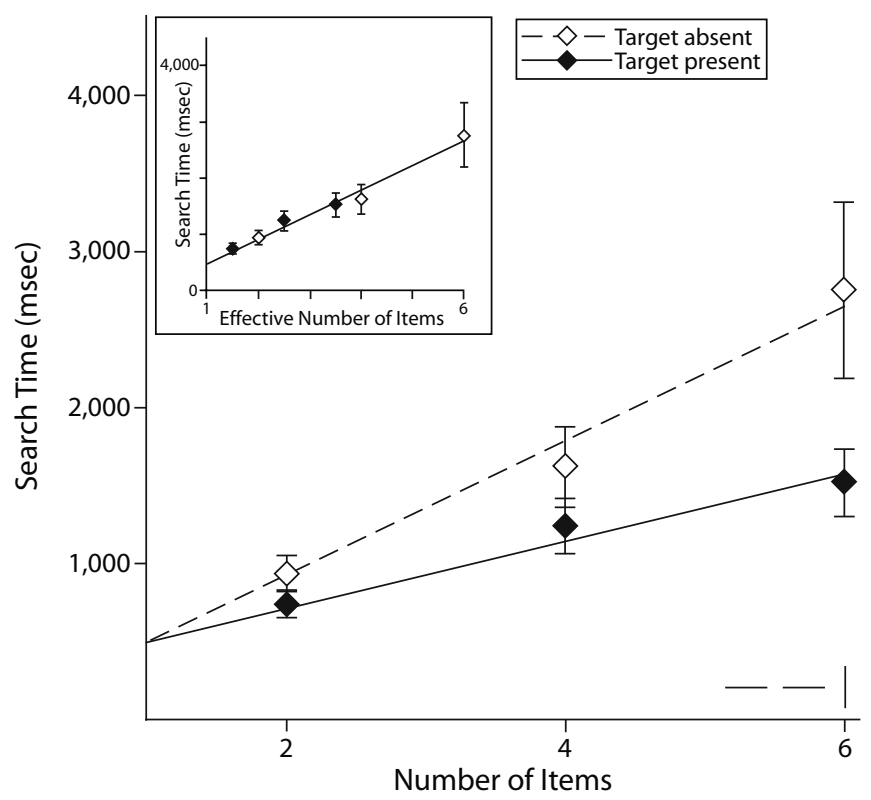

Figure 6. Haptic search times of Experiment 3. The slope (s) of the serial search model is $215 \pm 37$ msec per item. The intercept $\left(t_{1}\right)$ is $493 \pm 101 \mathrm{msec}$. See Figure 5 for further details. The target (vertical line) and distractors (horizontal lines) are shown at the bottom right. 
because parallel haptic search must be possible for clearly detectable targets among empty display elements.

\section{Method}

The same methods were used for measuring and analyzing the data as in Experiment 2, and the same participants once again participated. In this experiment, the target was a horizontal line and the distractors were blank pieces of ZY-TEX2 Swell paper. Note that although the distractors were empty, the number of fingers used and the number of possible target positions did differ between the blocks of trials.

\section{Results}

The results are shown in Figure 7 . We did not find a significant main effect for number of items but did find a main effect for target condition $(p<.05)$. We therefore fit the parallel search model to the data points, resulting in a value of $\bar{t}=516 \pm 138 \mathrm{msec}$ and a standard deviation $\sigma=376 \pm 199 \mathrm{msec}$. The fit was very good $\left(\chi^{2}=0.12, v=4, p<.005\right)$. The measured mean RT in the target-present condition was $512 \pm 66 \mathrm{msec}$, with a standard deviation of $296 \pm 55 \mathrm{msec}$. In this experiment, participants made even fewer errors than in the previous ones. The error rates were $0 \%$ in the two-item condition, $0.03 \%$ in the four-item condition, and $1.3 \%$ in the six-item condition.

\section{Discussion}

Because we did not find a main effect of the number of items and the fit of the parallel model was very good, we concluded that this task yielded a parallel search pattern as described in our model. The standard deviation derived from the model fit ( $376 \mathrm{msec}$ ) was close to the mean measured standard deviation in the target-present condition
(296 msec), indicating that our reasoning for the increase in RTs in the target-absent condition was justified.

When the display elements are empty, participants apparently do not have to check all fingers serially. This task could be considered a detection rather than a search task. The participants had to detect and localize a "bump" in a flat surrounding (an abrupt surface discontinuity, following Lederman \& Klatzky's, 1997, dimensions). In Experiments 2 and 3, participants had "bumps" under all fingertips that were being tested. The task in these experiments was to discriminate one of those items from the others on the basis of spatial features. In the present experiment, however, only one item was present that had such spatial features. The participants had to detect the item, but there was no need to compare it with other items.

The intercept of the haptic search function (the value for one item) was the same as in Experiment 3, in which the same element was used (a horizontal line). When one item is in the display, it apparently takes the same time to process it, regardless of the task. Thus, if the distinction we made in the previous paragraph is correct, we must conclude that the difference between detection and discrimination becomes clear only when there is more than one item in the display. This implies that the search for the feature of interest is really the serial process, rather than that limited resources exist for determining the orientation of a contour beneath a finger.

\section{EXPERIMENT 5}

In the present search experiments, the number of potential responses has covaried with the number of items in the display. According to the Hick-Hyman law (Hick, 1952;

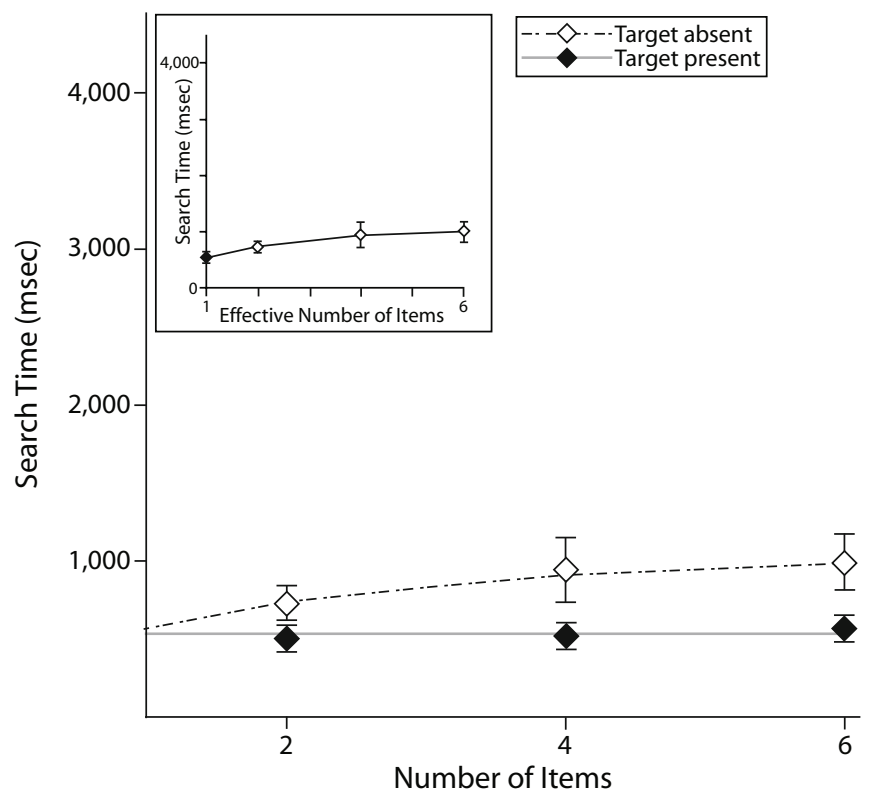

Figure 7. Haptic search times in Experiment 4. The lines represent the fit of the parallel search model to the data $(\bar{t}=516 \pm 138 \mathrm{msec}$ and $\sigma=$ $376 \pm 199$ msec; see the introduction and Figure 5 for further details). 
Hyman, 1953), this covariance could have influenced RTs: The more potential responses available, the longer the RT will be. The relation between the potential responses and the RT is described in the following equation, in which $n$ is the number of possible responses:

$$
\operatorname{RT}(n)=b \cdot \log _{2}(n+1) .
$$

In Experiment 4, we found no evidence for such a relationship in our data. However, in that experiment the relationship between stimulus and response was particularly easy, because participants had to lift the only finger that touched an item. To rule out the possibility that the serial search patterns in Experiments 2 and 3 were confounded by the combination of detecting the target and lifting the correct finger, we designed Experiment 5, in which we took the same target-distractor pair as in Experiment 2 (a cross as the target and circles as distractors), but the items were presented visually. It is well known that these items yield a parallel search pattern in vision (Shen et al., 2000). We placed the visual items close to the fingers and asked participants to move the finger near the target downward. If there was any effect of the number of items on the relationship between detecting the target and selecting the appropriate finger, this effect should lead to a significant slope in the target-present condition of this experiment.

\section{Method}

Participants. Eight participants, 2 male and 6 female, with a mean age of 27.9 years (range 25-30) participated in this experiment. They all had normal or corrected-to-normal vision, and 1 of them stated that he was left-handed. Two of these participants had also taken part in the haptic search experiments.

Procedure. Participants were seated behind a 12-in. TFT screen at a viewing distance of $45 \mathrm{~cm}$. A black fixation cross was shown on a white background for $500 \mathrm{msec}$, followed by a horizontal row of two, four, or six black items presented across the screen center. The background remained white during the whole experiment, and its brightness was $86 \mathrm{~cd} / \mathrm{m}^{2}$. The target was a cross, and the distractors were circles. The same absolute sizes and shapes of the items were used as in Experiment 2, and the distance between them was about $2 \mathrm{~cm}$. The participants sat with hands on the keyboard. Their task was to push the key corresponding to the target as soon as they saw the target. If no target was present, they had to push all keys. In the two-item condition, participants had their index fingers on the $g$ and the $h$ of the keyboard; in the four-item condition, their index and middle fingers were on the $f, g, h$, and $j$ keys; and in the six-item condition, their ring, middle, and index fingers were on the $d, f, g$, $h, j$, and $k$ keys. The participants were told that they should react as quickly as possible without making any errors. The experiment consisted of three blocks with two, four, or six items. Each block consisted of 48 trials, of which 12 (25\%) did not contain a target.

Analysis. We determined the median search time for each participant, condition, and number of items. Search time was defined as the time that elapsed from the moment the stimulus appeared on the screen until the moment that a button was pushed.

To test for effects of the number of items in the display and of target presence, we performed a repeated measures ANOVA with two factors: number of items (three levels) and target presence (two levels). We also fitted our parallel search model and the equation for the Hick-Hyman law to the data points.

\section{Results}

The search times are shown in Figure 8. We found a significant main effect of target presence $(p<.01)$ and an interaction between number of items and target presence $(p<.05)$. The parallel search model did not fit very well $\left(\chi^{2}=4.67, v=4, p<.25\right)$. The Hick-Hyman equation also gave a very bad fit to the data points $\left(\chi^{2}=5.30, v=4\right.$, $p<.25)$, so we fitted two separate lines instead. The fit for target-absent trials was $t(n)=492+18 n$, and the fit for target-present trials was $t(n)=390+37 n$.

\section{Discussion}

The results are in line neither with the parallel search model nor with the Hick-Hyman law. The fact that the slope of the search function in the target-absent condition was less steep than in the target-present condition is in line with the idea that in the target-absent condition there is only one possible response, whereas in the target-present condition the number of possible responses increases with increases in the number of items in the display.

However, when comparing the slopes of these functions (18 and $37 \mathrm{msec} /$ item) with the slopes of the search function from Experiments 2 and $3(290 \mathrm{msec} / \mathrm{item}$ and $215 \mathrm{msec} /$ item; see the inset in Figure 8), it is obvious that the serial search pattern that we found in the haptic experiments cannot be attributed to an increase in the number of possible responses when the number of items increases.

\section{GENERAL DISCUSSION}

These experiments show that our model can describe haptic search behavior very well. For serial search, the slope for target-absent conditions is twice as steep as that for target-present conditions. The search time when only one item is in the display is the same for the target-absent and target-present conditions. Searching for specific contours gives rise to serial search.

When searching for a target between empty elements, our experimental design was able to produce completely parallel haptic search. In that case, it was not necessary to check that the "right" target had been found; the aim was to search for an object, not for one of a particular kind.

The data clearly show that haptic search for a target that differs from the distractors in spatial features is necessarily serial. This is different from the results found in the visual domain, where a parallel search pattern is found for targets defined by various spatial features (Cavanagh, Arguin, \& Treisman, 1990; Linnell \& Humphreys, 2002; Mori \& Kataoka, 2004; Saarinen, 1995; Scialfa \& Joffe, 1998; Treisman \& Sato, 1990).

Other studies in the haptic domain have also found a serial pattern when using spatial features to distinguish between target and distractor (Lederman, Browse, \& Klatzky, 1988; Lederman \& Klatzky, 1997; Purdy, Lederman, \& Klatzky, 2004). However, the serial pattern in those studies was different from the pattern predicted for serial search by our model. In the introduction, we argued that this result could have been caused by the number of items having been varied across trials in the previous experiments. In Experiment 4, we showed that detecting and localizing a bump between empty stimulus elements is a parallel process: The number of empty elements was irrelevant. However, in a discrimination task, empty elements 


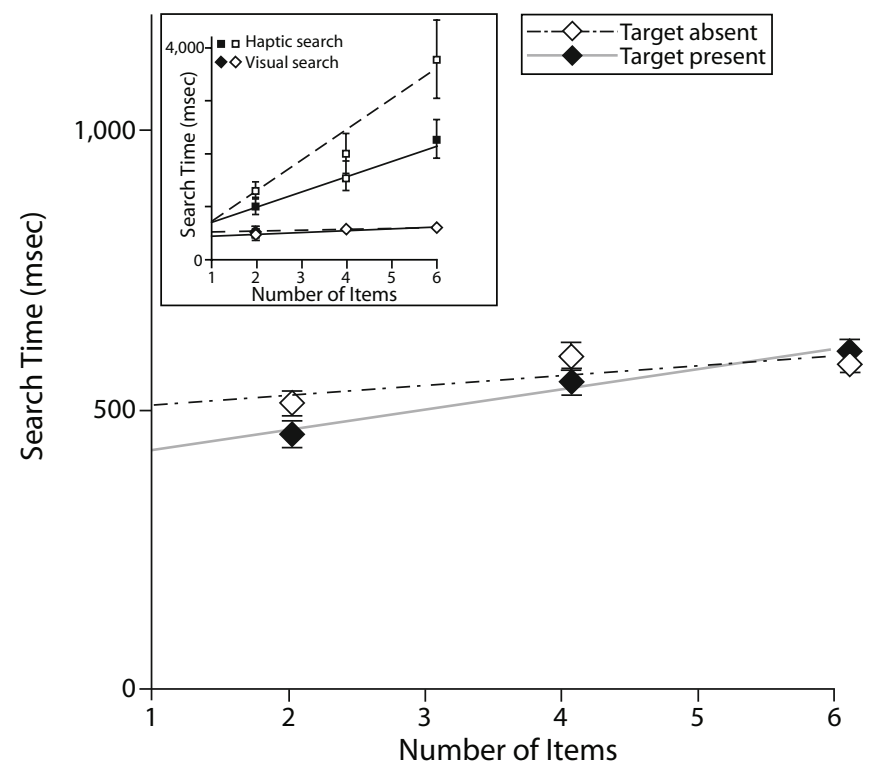

Figure 8. Search functions for visual search in Experiment 5. The lines represent separate fits for the data points when the target was absent and present. The points represent the means of the participants' median search times (with standard errors). The graph in the inset replots the data points of Experiments 2 and 5 at the same scale.

may not be as readily recognized as such. Alternatively, knowing which fingers may receive useful information may somehow guide a more efficient search, although comparing the search times in our Figures 2 (right column) and 6 does not suggest that this is the critical factor.

In summary, our search model described the data very well. In the experiments in which a spatial target had to be discriminated from spatial distractors, we found that the serial search model applied. In the experiment in which a spatial target had to be found among empty display elements, the parallel model applied.

\section{AUTHOR NOTE}

Correspondence relating to this article may be sent to K. E. Overvliet, Vrije Universiteit Amsterdam, Van der Boechorststraat 9, NL-1081 BT Amsterdam, The Netherlands (e-mail: krista.overvliet@gmail.com).

\section{REFERENCES}

Brown, J. M., Weisstein, N., \& May, J. G. (1992). Visual search for simple volumetric shapes. Perception \& Psychophysics, 51, 40-48.

Cavanagh, P., Arguin, M., \& Treisman, A. (1990). Effect of surface medium on visual search for orientation and size features. Journal of Experimental Psychology: Human Perception \& Performance, 16, 479-491.

Duncan, J., \& Humphreys, G. W. (1989). Visual search and stimulus similarity. Psychological Review, 96, 433-458.

Hick, W. E. (1952). On the rate of gain of information. Quarterly Journal of Experimental Psychology, 4, 11-26.

Hyman, R. (1953). Stimulus information as a determinant of reaction time. Journal of Experimental Psychology, 45, 188-196.

Joseph, J. S., Chun, M. M., \& NaKaYAMa, K. (1997). Attentional requirements in a "preattentive" feature search task. Nature, 387, 805-807.

Julesz, B. (1984). A brief outline of the texton theory of human vision. Trends in Neurosciences, 7, 41-45.

Julesz, B. (1986). Texton gradients: The texton theory revisited. Biological Cybernetics, 54, 245-251.
Lederman, S. J., Browse, R. A., \& Klatzky, R. L. (1988). Haptic processing of spatially distributed information. Perception \& Psychophysics, 44, 222-232.

LEDERMAN, S. J., \& KLATZKY, R. L. (1997). Relative availability of surface and object properties during early haptic processing. Journal of Experimental Psychology: Human Perception \& Performance, 23, 1680-1707.

LinNell, K. J., \& Humphreys, G. W. (2002). Visual search within and across dimensions: A case for within-dimension grouping. British Journal of Psychology, 93, 115-135.

Maioli, C., Benaglio, I., Siri, S., Sosta, K., \& Cappa, S. (2001). The integration of parallel and serial processing mechanisms in visual search: Evidence from eye movement recording. European Journal of Neuroscience, 13, 364-372.

Mori, S., \& Kataoka, A. (2004). Frame effects in visual search for line orientation. Perception \& Psychophysics, 66, 303-327.

NaKayama, K., \& Silverman, G. H. (1986). Serial and parallel processing of visual feature conjunctions. Nature, 320, 264-265.

PALMER, J. (1994). Set-size effects in visual search: The effect of attention is independent of the stimulus for simple tasks. Vision Research, 34, 1703-1721.

Press, W. H., Flannery, B. P., Teukolsky, S. A., \& Vetterling, W. T. (1992). Numerical recipes in C: The art of scientific computing (2nd ed.). Cambridge: Cambridge University Press.

Purdy, K. A., Lederman, S. J., \& Klatzky, R. L. (2004). Haptic processing of the location of a known property: Does knowing what you've touched tell you where it is? Canadian Journal of Experimental Psychology, 58, 32-45.

Quinlan, P. T., \& HumPhreYs, G. W. (1987). Visual search for targets defined by combinations of color, shape, and size: An examination of the task constraints on feature and conjunction searches. Perception \& Psychophysics, 41, 455-472.

SAARINEN, J. (1995). Visual search at different spatial scales. Scandinavian Journal of Psychology, 36, 1-9.

Scialfa, C. T., \& Joffe, K. M. (1998). Response times and eye movements in feature and conjunction search as a function of target eccentricity. Perception \& Psychophysics, 60, 1067-1082.

Shen, J., Reingold, E. M., \& Pomplun, M. (2000). Distractor ratio influences patterns of eye movements during visual search. Perception, 29, 241-250. 
Treisman, A. M., \& Gelade, G. (1980). A feature-integration theory of attention. Cognitive Psychology, 12, 97-136.

Treisman, A. M., \& Gormican, S. (1988). Feature analysis in early vision: Evidence from search asymmetries. Psychological Review, 95 15-48.

Treisman, A. M., \& Sato, S. (1990). Conjunction search revisited. Journal of Experimental Psychology: Human Perception \& Performance, 16, 459-478.

Wolfe, J. M. (1994). Guided Search 2.0: A revised model of visual search. Psychonomic Bulletin \& Review, 1, 202-238.

Wolfe, J. M., CAVE, K. R., \& Franzel, S. L. (1989). Guided search: An alternative to the feature integration model for visual search. Journal of Experimental Psychology: Human Perception \& Performance, 15, 419-433.

Wolfe, J. M., \& Horowitz, T. S. (2004). What attributes guide the deployment of visual attention and how do they do it? Nature Reviews Neuroscience, 5, 495-501.

\section{NOTE}

1. Equation 4 is based on the cumulative normal distribution $(C N D)$ :

$$
\begin{aligned}
C N D(x) & =\int_{-\infty}^{x} \frac{1}{\sigma \sqrt{2 \pi}} e^{-(x-\mu)^{2} /\left(2 \sigma^{2}\right)} \\
& =\frac{1}{2}\left(1+\operatorname{erf} \frac{x-\mu}{\sigma \sqrt{2}}\right)
\end{aligned}
$$

If we want to find the median value of a normally distributed data set of RTs, we need to take the value for which the $C N D$ is $0.5[C N D(x)=$ 0.5 ]. If we want to know the median of the highest RT drawn from $n$ distributions, we need to take the value for which the product of those $n$ distributions equals $0.5\left[C N D(x)^{n}=0.5\right]$.

(Manuscript received September 21, 2005; revision accepted for publication February 15, 2007.) 